\title{
J-Shaped relationship between habitual coffee consumption and 10-year (2002-2012) Cardiovascular Disease incidence: the ATTICA Study
}

Georgia-Maria KOULI ${ }^{1}$, MSc, Demosthenes B. PANAGIOTAKOS ${ }^{1}$, PhD, Ekavi N GEORGOUSOPOULOU ${ }^{1,2}$, PhD, Duane D. MELLOR ${ }^{2}$, PhD, Christina CHRYSOHOOU ${ }^{3}, \mathrm{MD}, \mathrm{MSc}$, PhD, Adela ZANA ${ }^{1}, \mathrm{MSc}^{\prime}$, Constantine TSIGOS $^{1}, \mathrm{MD}, \mathrm{PhD}$, Dimitrios TOUSOULIS ${ }^{3}, \mathrm{MD}, \mathrm{PhD}$, Christodoulos STEFANADIS ${ }^{3}, \mathrm{MD}$, PhD, Christos PITSAVOS ${ }^{3}, M D$, PhD.

${ }_{1}$ Department of Nutrition and Dietetics, School of Health Science and Education Harokopio University, Athens, Greece; ${ }^{2}$ Faculty of Health, University of Canberra, Canberra, Australian Capital Territory, Australia; ${ }^{3}$ First Cardiology Clinic, School of Medicine, University of Athens, Greece; All authors state that they take the responsibility for all aspects of the reliability and freedom from bias of the data presented and their discussed interpretation. Corresponding author
Professor Demosthenes B. Panagiotakos 46 Paleon Polemiston St., Glyfada, Attica, 16674 , Greece Tel. +30 210-9549332, \& $+30210-9600719$ (Fax)
E-mail. d.b.panagiotakos@usa.net

\section{ABSTRACT}

Purpose: The purpose of this work was to evaluate the association between coffee consumption and 10-year cardiovascular disease (CVD) incidence in the ATTICA Study, and whether this is modified by baseline the presence or absence of-metabolic syndrome (MetS) at baseline. Methods: During 20012002, 3042 healthy adults (1514 men and 1528 women) living in the greater area of Athens were voluntarily recruited to the ATTICA study. In 2011-2012, the 10-year follow-up was performed in 2583 participants (15\% of the participants were lost to followup). Coffee consumption was assessed by a validated food-frequency questionnaire at baseline (abstention, low, moderate, heavy). Incidence of fatal or non-fatal CVD event was recorded using WHO-ICD-10 criteria and MetS was defined by the National Cholesterol Education Program Adult Treatment panel III (revised) criteria. Results: Overall, after controlling for potential CVD risk factors, the multivariate analysis revealed a J-shaped association between daily coffee drinking and the risk for a first CVD event in a 10-year period. Particularly, the odds ratio for low $(<150 \mathrm{ml} / \mathrm{d})$, moderate $(150-250 \mathrm{ml} / \mathrm{d})$ and heavy coffee consumption (>250 $\mathrm{ml} / \mathrm{d})$, compared to abstention, were $0.44(95 \% \mathrm{CI}$ : $0.29-0.68), 0.49$ (95\% CI: $0.27-0.92)$ and 2.48 (95\%CI: $1.56-1.93)$ respectively. This inverse association was also verified among participants without MetS at baseline but not among participants with the MetS. Conclusions: These data supports the protective effect of drinking moderate quantities of coffee (equivalent to approximately $1-2$ cups daily) against CVD incidents. This protective effect was only significant for participants without MetS at baseline. Key words: Cardiovascular Diseases; Metabolic Syndrome X; coffee; inflammation

\section{Introduction}

Coffee is one of the most commonly consumed beverages around the world. Because of its popularity, investigating the association between coffee consumption and chronic disease risk has important public health consequences. Coffee is a complex chemical mixture, containing $>1000$ bioactive compounds with potential beneficial properties, including insulin-sensitizing and antiinflammatory effects $[1,2]$. Notably, chronic low-grade inflammation underlies the pathogenesis of all components of metabolic syndrome (MetS): hyperglycemia, insulin resistance, hypertension, obesity, and dyslipidemia, all of which are risk factors for cardiovascular disease. Therefore, dietary interventions designed to reduce the inflammatory procedure could be of benefit to reduce the cardiovascular disease (CVD) risk [3]. Existing data do support the effect of habitual coffee consumption on cardiovascular health.

Since 2000, the association between coffee consumption and other CVD outcomes including stroke, heart failure, and total CVD mortality has been widely investigated. However, the nature of this association remains unclear since many prospective cohort studies reported conflicting data. A recent cohort study by Liu et al found that coffee consumption (4 cups/day) was associated with increased mortality, but the association was no longer significant for persons under 55 years old [4], whereas the EPIC study reported that coffee consumption was not associated with risk of CVD [5]. Interestingly, in 2014, a meta-analysis of 21 prospective studies and 997,464 participants (121,915 deaths), found a non-linear dose-response relationship between coffee and CVD mortality suggesting that the largest risk reductions were observed for 3 cups/day for CVD mortality (21\% lower risk) [2]. These results for coffee-CVD mortality were comparable to those reported in a previous meta-analysis including 36 studies and 1,279,804 participants (36,352 CVD cases) that strengthened the coffee-CVD non-linear U-shaped relationship, i.e. moderate coffee consumption (3-5 cups/day) was associated with lower CVD risk, and heavy coffee consumption ( $\geq 6$ cups/day) was neither associated with a higher nor a lower risk of CVD [6].

While current research aims to reveal the actual association between coffee consumption and CVD risk little is known about the potential mediators of this association. Prolonged inflammatory processes is closely related to oxidative stress and contribute to the pathogenesis of atherosclerosis $[7,8]$. Epidemiological data have found that coffee is associated with reduced biomarkers of oxidative stress 
since several compounds found in coffee (polyphenols i.e. chlorogenic acid; volatile aroma compounds e.t.c.) contribute to its antioxidant capacity; moreover, several studies have revealed a beneficial, acute effect of coffee consumption on endothelial function $[9,10]$. As a result, coffee drinking increases plasma antioxidants that can antagonize the oxidative stress and inflammation process, which both underlie atherogenesis and increase aortic stiffness.

Despite the increasing understanding of the emerging role of chronic inflammation in CVD has stimulated research into the potential protective effect of coffee consumption, a major source of dietary antioxidants, the evaluation of inflammatory and oxidative stress indices on coffee-CVD relationship in the long-term has rarely been studied. Furthermore, very few studies have tested the aforementioned association at high CVD risk people, e.g., hypertensive, diabetic [3, 11]. Therefore, the aim of the current work was to prospectively examine the associations of coffee consumption and the 10-year CVD incidence in the participants of the ATTICA study, and examine whether this is modified by chronic inflammation and oxidative stress status. Secondly, we wished to assess whether this association was affected by the presence of MetS among apparently healthy adults. By answering the tested hypotheses new insights in the coffee consumption - CVD risk associations will be revealed.

\section{Materials and Methods}

\subsection{Sampling procedure at baseline examination}

The ATTICA Study is a prospective population-based study that was carried out in the greater metropolitan area of Athens in Greece, which included $78 \%$ urban and $22 \%$ rural population. The baseline examination of the study was carried out during 2001-2002 [12]. The sampling procedure anticipated enrolling only one participant per household; it was random, multistage and based on the age, sex distribution of the Attica region (census of 2001). Of the 4056 invited individuals at baselineexamination, 3042 agreed to participate (75\% participation rate); 1514 of the participants were men (18-87 years) and 1528 were women (18-89 years). All participants were interviewed by trained personnel (cardiologists, general practitioners, dieticians and nurses) who used a standard questionnaire. Exclusion of CVD at baseline evaluation was performed through a detailed clinical evaluation by the physicians of the study. The examination was performed in the individuals' homes or workplaces.

\subsection{Baseline measurements}

The baseline evaluation included information about demographic characteristics (age, sex), personal history of hypertension, hypercholesterolemia and diabetes, dietary and other lifestyle habits (i.e., smoking status, physical activity, education). In the present analysis, smoking was defined through years of smoking while education was measured in years of school. The evaluation of the dietary habits was based on a validated semi-quantitative food-frequency questionnaire [13], the EPIC-Greek questionnaire that was kindly provided by the Unit of Nutrition of Athens Medical School. On the basis of the FFQ, all participants were asked about their usual frequency (average) of daily coffee consumption. 


\begin{tabular}{|c|c|c|c|c|c|}
\hline & \multicolumn{4}{|c|}{ Daily coffee consumption (ml/d) } & \multirow[t]{2}{*}{$\boldsymbol{P}^{1}$} \\
\hline & $\begin{array}{c}\text { Never } \\
\mathbf{0} \\
(n=342)\end{array}$ & $\begin{array}{c}\text { Low } \\
0-150 \\
(n=1126)\end{array}$ & $\begin{array}{c}\text { Moderate } \\
150-250 \\
(n=338)\end{array}$ & $\begin{array}{c}\text { High } \\
>\mathbf{2 5 0} \\
(n=214)\end{array}$ & \\
\hline 10 -year incidence of CVD, \% & 19 & 16 & 7 & 25 & $<0.001$ \\
\hline \multicolumn{6}{|c|}{ Demographic, lifestyle and clinical characteristics } \\
\hline Age (years) & $50 \pm 14$ & $46 \pm 13$ & $36 \pm 12$ & $50 \pm 13$ & $<0.001$ \\
\hline Gender, \% male & 26 & 45 & 66 & 77 & $<0.001$ \\
\hline Smoking (years) & $20 \pm 11$ & $20 \pm 12$ & $16 \pm 10$ & $23 \pm 11$ & $<0.001$ \\
\hline Physical activity, \% & 36 & 40 & 41 & 39 & 0.38 \\
\hline Waist to hip ratio & $0.83 \pm 0.09$ & $0.86 \pm 0.11$ & $0.86 \pm 0.10$ & $0.92 \pm 0.12$ & $<0.001$ \\
\hline Education (years of school) & $12 \pm 3$ & $12 \pm 4$ & $13 \pm 3$ & $12 \pm 4$ & $<0.001$ \\
\hline Hypertension, \%yes & 29 & 31 & 21 & 38 & $<0.001$ \\
\hline Diabetes Mellitus, \%yes & 7 & 8 & 3 & 10 & $<0.001$ \\
\hline Hypercholesterolemia, \%yes & 52 & 37 & 29 & 55 & $<0.001$ \\
\hline MedDietScore (range 0-55) & $25 \pm 6$ & $26 \pm 7$ & $27 \pm 7$ & $24 \pm 6$ & $<0.001$ \\
\hline Metabolic Syndrome, \%yes & 21 & 21 & 14 & 24 & $<0.001$ \\
\hline \multicolumn{6}{|c|}{ Oxidative and Inflammatory biomarkers } \\
\hline Oxidised LDL-C (mg/dL) & $72 \pm 21$ & $49 \pm 23$ & $63 \pm 30$ & $83 \pm 22$ & $<0.001$ \\
\hline Interleukin-6 (ng/mL) & $1.5 \pm 0.7$ & $1.5 \pm 0.5$ & $1.4 \pm 0.3$ & $1.5 \pm 0.8$ & $<0.001$ \\
\hline C-reactive protein $(\mathrm{mg} / \mathrm{L})$ & $2.1 \pm 2.5$ & $2 \pm 2.5$ & $1.8 \pm 2.3$ & $1.8 \pm 2.1$ & 0.15 \\
\hline Homocysteine $(\mu \mathrm{mol} / \mathrm{L})$ & $11 \pm 4$ & $12 \pm 6$ & $12 \pm 7$ & $13 \pm 5$ & 0.03 \\
\hline Fibrinogen $(\mathrm{mg} / \mathrm{dL})$ & $331 \pm 78$ & $314 \pm 69$ & $288 \pm 61$ & $319 \pm 67$ & $<0.001$ \\
\hline
\end{tabular}

The MedDietScore was also applied (range 0-55) to evaluate adherence to the Mediterranean diet [14]. For the ascertainment of physical activity status the International Physical Activity Questionnaire was used (IPAQ) [15], as an index of weekly energy expenditure using frequency (times per week), duration (in minutes per time) and intensity of sports or other habits related to physical activity (in expended calories per time). Participants who did not report any physical activities were defined as physically inactive. Waist circumference was measured in the middle between the lowest rib and the iliac crest using an inelastic measuring tape to the nearest $0.5 \mathrm{~cm}$, while waist to hip ratio was also calculated. Arterial blood pressure (mean of 3 recordings) was measured at the end of the baseline physical examination in a sitting position after resting for at least 30 minutes. Participants whose average blood pressure levels were greater or equal to $140 / 90 \mathrm{mmHg}$ or were under antihypertensive medication were classified as having hypertension. Blood samples were collected from the antecubital vein between 8 to $10 \mathrm{am}$, in a sitting position after 12 hours of fasting and alcohol abstinence. Blood lipids (total cholesterol, HDL-cholesterol) and triglycerides were measured using chromatographic enzymic method in a Technicon automatic analyser RA-1000 (Dade Behring, Marburg, Germany). Hypercholesterolemia was defined as total cholesterol levels greater than $200 \mathrm{mg} / \mathrm{dl}$ or the use of lipids lowering agents. Blood glucose levels $(\mathrm{mg} / \mathrm{dl})$ were measured with a Beckman Glucose Analyzer (Beckman Instruments, Fullerton, CA, USA). Diabetes mellitus (type 2) was defined according to the American Diabetes Association diagnostic criteria (i.e., blood glucose levels greater than $125 \mathrm{mg} / \mathrm{dl}$ classified participants as having diabetes) [16]. Participants were classified as having the MetS or not, according to the NCEP ATP III (revised 2005) definition. MetS presence was defined if three or more of the following metabolic syndrome components were present: waist circumference $\geq 102 \mathrm{~cm}$ for males or $\geq 88 \mathrm{~cm}$ for females; triglyceride level $\geq 150 \mathrm{mg} / \mathrm{dl}$; HDL cholesterol level $<40 \mathrm{mg} / \mathrm{dL}$ for males or $<50 \mathrm{mg} / \mathrm{dL}$ for females; blood pressure $\geq 130 / 85 \mathrm{mmHg}$; fasting blood glucose $\geq 100 \mathrm{mg} / \mathrm{dL}$ [17].

High sensitivity C-reactive protein was assayed by particle-enhanced immunonephelometry (N Latex, Dade-Behring Marburg GmbH, Marburg, Germany). Participants with chronic inflammation or CRP levels above $10 \mathrm{mg} / \mathrm{L}$ were not included in the analyses. Furthermore, inflammatory markers, such as homocysteine, fibrinogen, Interleukin-6 (IL-6)), and oxidative stress markers (plasma oxidized LDL-cholesterol (ox-LDL)), were also measured.

\subsection{Coffee consumption}

All participants were asked about average coffee drinking habits during the preceding year. Consumption of all reported types of coffee (i.e., instant coffee, brewed coffee, Greek-type coffee, cappuccino or filtered coffee) were recorded in cups of coffee per day $(1 \mathrm{cup}=150 \mathrm{ml})$ and then recalculated (in $\mathrm{ml}$ ), after adjustment for $28 \%$ caffeine containment [18] for analytical reasons. Thus, after this re-calculation, 1 "adjusted" cup of coffee (i.e., $150 \mathrm{ml}$ ) could be equivalent to $450 \mathrm{ml}$ 
brewed coffee or $300 \mathrm{ml}$ instant coffee. According to the observed distribution of coffee drinking pattern, four coffee consumption categories were created: abstention, low (0-150 ml/day), moderate (150-250 ml/day) and high (>250 ml/day). Information of decaffeinated coffee drinking was also recorded, but not used in the analysis because of the very small number of participants reported drinking this type of coffee $(n=47)$.

\subsection{Follow-up examination (2011-2012)}

During 2011-12, the ATTICA Study's investigators performed the 10-year follow-up (mean follow-up time 8.41 y) [19]. Of the $n=3042$ initially enrolled participants, $n=2583$ were found during the follow-up ( $85 \%$ participation rate). Mean age at baseline ( \pm SD) was $45 \pm 14$ and $46 \pm 14$ years for women and men respectively (no difference with the overall sample). Of the individuals that were lost to follow-up (i.e., $n=459$ ), $n=224$ were not found because of missing or wrong addresses and telephone numbers that they have provided at baseline examination and $n=235$ because they denied being re-examined. Regarding CVD evaluation at follow-up clinically accurate data were obtained from $n=2020$ participants. In order to participate in the follow-up all participants were initially contacted through telephone calls. Afterwards, the investigators approached the $n=2583$ participants that were allocated in the follow-up and performed a detailed evaluation of their medical records. In particular, from June 2011 to June 2012 information about participants': (a) vital status (death from any cause or due to CVD), (b) development of CHD (i.e., myocardial infarction, angina pectoris, other identified forms of ischemia -WHO-ICD coding 410-414.9, 427.2, 427.6-, heart failure of different types, and chronic arrhythmias -WHO-ICD coding 400.0-404.9, 427.0 -427.5, 427.9-), (c) development of stroke (WHO-ICD coding 430-438). The working sample size was adequate to achieve $92 \%$ statistical power to evaluate relative risk of 0.70 between the null and the alternative two-sided hypothesis, when the exposure variable (i.e., coffee drinking category) was increased by 1 -unit and with a significance level (alpha) of 0.05

\subsection{Bioethics}

The study was approved by the Bioethics Committee of Athens Medical School and was carried out in accordance to the Declaration of Helsinki (1989) of the World Medical Association. Prior to the collection of any information, participants were informed about the aims and procedures of the study and provided their written signed consent.

\subsection{Statistical analysis}

Crude, non-fatal and fatal incidence rates of combined CVD (i.e., CHD or stroke) were calculated as the ratio of new cases to the number of people participated in the follow-up. Incidence by coffee drinking status was calculated as the ratio of new cases in each coffee category to the number of participants in the same category. The continuous variables were tested for normality through P-P plots and presented as mean values \pm standard deviation or as median $\left(1^{\text {st }}, 3^{\text {rd }}\right.$ quartile) if not normally distributed while categorical variables were presented as relative frequencies. Associations between categorical variables were tested using the chi-square test, while between continuous variables using Pearson $r$ or Spearman's rho coefficients, for the normally and skewed variables respectively. Comparisons of mean values of normally distributed variables by coffee drinking status were performed using analysis of variance (ANOVA) and post-hoc analyses using Bonferroni rule were performed to account for the inflation of the probability of type-I error. Comparisons between mean values of normally distributed variables between those who developed an event and the rest of the participants were performed using Student's t-test, after controlling for equality of variances using the Levene's test. Comparisons of continuous variables that did not follow the normal distribution were performed using the non-parametric U-test proposed by Mann and Whitney and the Kruskal-Wallis $\mathrm{H}$ test. The hazard ratios (HR) and the corresponding 95\% confidence intervals (CI) of developing a CVD event during the 10-year period, according to the participants' baseline characteristics were estimated using Cox proportional hazards models. The time to CVD event was recorded on annual basis. Furthermore, non-linear trend analysis was applied in order to evaluate curve fitting on the effect size measures (hazard ratios) between coffee drinking categories and the outcome. Trend analysis was applied by fitting smoothing lines (linear, quadratic or cubic) on the odds ratios derived by age-sex adjusted analysis, by coffee drinking category (coffee was categorized in 4 categories to perform this analysis, abstention, 0-150 $\mathrm{ml}, 150-250 \mathrm{ml},>250 \mathrm{ml}$ ); the corresponding R-squared values indicated which line best fit the observed data. All reported $p$-values are based on two-sided tests. SPSS version 21 (Statistical Package for Social Sciences, SPSS Inc, Chicago, IL, U.S.A.) software was used for all the statistical calculations.

\section{Results}

\subsection{Ten-year CVD incidence}

The 10 -year fatal or non-fatal CVD event rate was 157 cases/ 1000 participants $(n=317)$, 198 being in males (198 cases/ 1000 participants) and 119 being in females (117 cases/ 1000 participants) ( $p$ for gender difference $<0.001)$.

Table 2. Results from the multi-adjusted Cox proportional hazards models that were developed to evaluate the $10-$
year risk of having a cardiovascular event (outcome) according to the daily coffee consumption among ATTICA
study participants $(\mathrm{n}=2020)$.
\begin{tabular}{|l|c|c|c|}
\hline All participants & Hazard Ratios (HR), 95\% Confidence Intervals \\
\hline & Model 1 & Model 2 & Model 3 \\
\hline
\end{tabular}




\begin{tabular}{|l|c|c|c|}
\hline Age (per 1 year) & $1.01(1.08-1.10)$ & $1.07(1.05-1.10)$ & $1.06(1.04-1.09)$ \\
\hline Men vs. Women & $2.13(1.59-2.86)$ & $2.01(1.19-3.40)$ & $1.99(1.16-3.42)$ \\
\hline Coffee drinking & & & \\
\hline \multicolumn{1}{|c|}{ Low coffee drinkers vs. abstainers } & $0.47(0.32-0.71)^{2}$ & $0.47(0.31-0.72)^{2}$ & $0.44(0.29-0.68)^{2}$ \\
\hline Moderate coffee drinkers vs. abstainers & $0.58(0.31-1.04)^{2}$ & $0.53(0.29-0.97)^{2}$ & $0.49(0.27-0.92)^{2}$ \\
\hline \multicolumn{1}{|c|}{ High coffee drinkers vs. abstainers } & $2.81(1.84-4.23)^{2}$ & $2.62(1.67-4.09)^{2}$ & $2.48(1.56-1.93)^{2}$ \\
\hline Smokers vs. non smokers & - & $1.00(0.98-1.02)$ & $1.00(0.98-1.02)$ \\
\hline Physically activity vs. inactive & - & $0.68(0.45-1.02)$ & $0.68(0.45-1.04)$ \\
\hline Waist to hip ratio & - & $8.7(0.95-80)$ & $3.86(0.38-39)$ \\
\hline Education (years of school) & - & $0.97(0.92-1.02)$ & $0.98(0.92-1.03)$ \\
\hline MedDietScore (range 0-55) & - & $0.96(0.93-1.001)$ & $0.96(0.93-0.99)$ \\
\hline Hypertension $(\mathrm{y} / \mathrm{n})$ & - & - & $1.13(0.74-1.70)$ \\
\hline Diabetes Mellitus $(\mathrm{y} / \mathrm{n})$ & - & - & $2.4(1.33-4.40)$ \\
\hline Hypercholesterolemia $(\mathrm{y} / \mathrm{n})$ & - & - & $1.42(0.95-2.13)$ \\
\hline
\end{tabular}

\subsection{Participants' baseline characteristics by coffee drinking status}

Baseline characteristics of the studied sample, by adjusted coffee drinking status ( 0 , $<150 \mathrm{ml} / \mathrm{d}, 150-250 \mathrm{ml} / \mathrm{d},>250 \mathrm{ml} / \mathrm{d}$ ), are presented in Table 1. As it can be seen, compared to abstainers, the group of participants, who were drinking daily lower amounts of coffee up to 1 adjusted cup ( $<150 \mathrm{ml} / \mathrm{d}$ ), included younger men, with increased waist to hip ratio, with increased arterial blood pressure and glucose, and decreased levels of blood lipids, who adhered closer to the Mediterranean diet, (all p-values <0.001). Similarly, subjects drinking 1 to 2 adjusted cups of coffee (150-250 ml/d), compared to coffee non-drinkers, were more likely to be younger male participants, lighter smokers, more strongly adhering to the Mediterranean diet (i.e. higher MedDietScore, mean score of 27/55), but also less likely to have a history of hypertension, diabetes, hypercholesterolemia and therefore MetS. Finally, those reporting drinking over 2 adjusted cups of coffee daily (>250 $\mathrm{ml} / \mathrm{d}$ ) when compared to abstainers were the most likely to be male, heavier smokers, obese, lower adherers to the Mediterranean diet, with increased susceptibility to hypertension, diabetes mellitus, hypercholesterolemia and MetS. No significant differences were observed out as regards physical activity levels $(p=0.38)$.

Mean circulating concentrations of participants' inflammation state characteristics, by coffee consumption categories, are also shown in Table 1. Overall, participants who consumed low and moderate amounts of coffee daily (barely 1-2 cups of coffee) had on average lower ox-LDL, lower fibrinogen but higher homocysteine concentrations, compared to coffee abstainers. Similar results were extracted for fibrinogen and homocysteine levels for heavy coffee drinkers, consuming over 2 cups of coffee daily, but not for ox-LDL levels which were increased. As for the rest of the inflammatory biomarkers, IL- 6 was comparable among the 4 groups, while CRP levels did not differ significantly by coffee drinking status $(p=0.15)$.

\subsection{0-year CVD incidence and coffee drinking}

The 10 -year incidence of CVD (Table 1) was $n=60(19 \%)$ among coffee abstainers, $n=175$ $(16 \%)$ among participants who consumed lower amounts of coffee, $n=23(7 \%)$ among moderate drinkers and $n=54(25 \%)$ among heavy coffee drinkers $(p<0.001)$. Based on these rates a trend analysis was applied; a J-shape association was revealed ( $\mathrm{p}$ for parabolic trend $=0.05$ ).

\begin{tabular}{|c|c|c|c|}
\hline & \multicolumn{3}{|c|}{ Hazard Ratios $(\mathrm{HR})^{2}, 95 \%$ CI for coffee consumption } \\
\hline & Low vs. Never & Moderate vs. Never & High vs. Never \\
\hline Full Model ${ }^{1}$ & $0.44(0.29-0.68)$ & $0.49(0.27-0.92)$ & $2.48(1.56-1.93)$ \\
\hline Full Model ${ }^{1}+C$-reactive protein & $0.46(0.24-0.89)$ & $0.42(0.18-0.98)$ & $1.60(0.74-3.46)$ \\
\hline Full Model ${ }^{1}+$ Oxidised $L D L-C$ & $0.34(0.12-0.95)$ & $0.19(0.05-0.83)$ & $1.26(0.46-3.46)$ \\
\hline Full Model ${ }^{1}+$ Interleukin-6 & $0.49(0.25-0.95)$ & $0.43(0.18-0.99)$ & $1.62(0.76-3.45)$ \\
\hline Full Model ${ }^{1}+$ Homocysteine & $0.57(0.24-0.98)$ & $0.50(0.18-0.97)$ & $1.76(0.66-4.72)$ \\
\hline Full Model ${ }^{1}+$ Fibrinogen & $0.42(0.21-0.82)$ & $0.32(0.13-0.75)$ & $1.56(0.69-3.51)$ \\
\hline
\end{tabular}

However, all the afore mentioned comparisons may be prone to bias due to potential factors; therefore multi-adjusted analysis was performed, controlling for various CVD covariates (see Table 2). Age-sex adjusted analysis revealed a significant association between coffee drinking and CVD incidence (Table 2, Model 1); and a J-shape trend was evident $(p<0.05)$. When personal and behavioral characteristics were taken into consideration (i.e., waist to hip circumference ratio, ever smoking, physical activity status, education and adherence to the Mediterranean diet), drinking 1-2 cups of coffee/d was inversely associated with CVD incidence compared to abstention, whereas drinking $>2$ cups of coffee daily was associated with increased risk of CVD events (Table 2, Model 2); still J-shape trend was evident even after adjusting for the aforementioned lifestyle behaviors. The aforementioned findings regarding the J-shaped trend of coffee drinking on CVD risk remained significant even after controlling for the classical CVD risk factors (i.e., hypertension, diabetes, hypercholesterolemia) (Table 2, Model 3). Moreover, and in order to further take into account the 
role of smoking, a restricted analysis only among never smokers was applied and revealed a similar trend of coffee drinking on CVD risk (HR, 95\%CI for abstainers/low/moderate/high coffee drinkers: 1 (Reference), $0.46(0.27,0.77), 0.49(0.21,1.18)$ and $3.15(1.86,5.14)$, respectively). Coffee intake might also be confounded by specific dietary habits, i.e., intakes of fruits, juices and sugar sweetened beverages; additional analysis based on Model 3 (Table 2) showed that the J-shaped trend of coffee drinking on CVD risk remained significant even after controlling for the aforementioned covariates (HR, 95\%CI for abstainers/low/moderate/high coffee drinkers: 1 (Reference), $0.48(0.32,0.73), 0.62$ $(0.34,1.11)$ and $2.85(1.86,4.38)$, respectively). Additionally, taking into account that the ATTICA study had previously shown a dose-response relation between coffee consumption and inflammatory markers (C-reactive protein, interleukin-6, oxidative - LDL, homocysteine) [20], the latter biomarkers were entered consecutively and separately in the latest fully adjusted Model 3; no alterations as regards the observed J-shaped trend of coffee drinking on CVD risk were found.

Notably, a significant interaction was observed between coffee drinking and presence of MetS on 10-year CVD risk ( $\mathrm{p}$ for interaction $=0.012$ ). This was also supported by the significant association observed between quantity of coffee drinking and prevalence of MetS (Table 1). Thus, the analysis was repeated by MetS group at the baseline examination. Based on the additional stratified analysis, it was observed that low and moderate daily coffee consumption compared to coffee abstention remained significantly protective against CVD risk among participants who did not have MetS at baseline (OR for $0-150 \mathrm{ml}$ daily vs. abstainers $=0.26 ; 95 \% \mathrm{CI}: 0.16,0.54$, HR for $150-250 \mathrm{ml}$ daily vs. abstainers $=0.18 ; 95 \% \mathrm{CI}: 0.06,0.52)$; however coffee consumption was not associated with 10 -year CVD risk among participants with MetS.

\section{Discussion}

The present study demonstrated a J-shaped association between coffee consumption and the 10-year CVD incidence. In particular, low and moderate coffee consumption (150-250 ml coffee/d), was associated with $56 \%$ and $51 \%$ lower CVD risk respectively, compares to abstention, whereas heavy consumption ( $>250 \mathrm{ml}$ coffee/d) was associated with a significant 2.5 -fold increase in risk, independently of the known potential confounders including lifestyle factors and clinical characteristics. However, in an effort to identify potential mediators of the above mentioned association we failed to identify any of the known inflammatory biomarkers as having such a mediating effect. The protective effect of consuming daily 1-2 cups of coffee daily was significant in the overall sample and among MetS free participants but not in high-CVD risk individuals (i.e MetS subjects), indicating that atherosclerotic mechanisms have already been triggered in earlier stages of their life and have, thus possibly neutralizing the protective effect of coffee on CVD risk [21-23]. In any case, the reported findings offer a considerable message for public health i.e. long-term low and moderate daily coffee drinking may be of benefit on 10-year risk for CVD, whereas heavy consumption is associated with significantly increased risk in healthy adults.

A potential biological mechanism for the inverse association of low and moderate coffee consumption with 10-year CVD incidence may be related, according to recent studies, to the fact that coffee is a source of antioxidants that may influence oxidative stress and endothelium function $[3,24]$. An imbalance of antioxidant defences and the production of reactive oxygen species can contribute to the pathogenesis and progression of atherosclerosis via various pathways [25, 26]. During inflammation, the produced, by active NADPH oxidase, superoxide $\left(\mathrm{O}_{2}{ }^{-}\right)$leads to other reactive oxygen species. Apart from the respiratory burst, mitochondrial electron transport chain during inflammation produces extra $\mathrm{O}_{2}{ }^{-}$that generates oxidative stress [27]. Chlorogenic acid (CGA) is the most prevalent polyphenol in coffee and has high bioavailability. Moreover, recent data support that polyphenols offer protection by stimulating endogenous antioxidant defence systems through specific gene response elements that are inducible by oxidative stress $[28,29]$. In the context, CGA, the most highly prevalent polyphenol in coffee, may play a key role in acutely raising the plasma antioxidant capacity in healthy adults, thereby lowering the risk of atherosclerosis [30, 31].

The observed J-shaped association between coffee consumption and CVD risk (i.e., low/moderate consumption seems beneficial, whereas increased consumption may be harmful) can also be based on other plausible biological mechanisms. In particular, coffee is a composite brew with several bioactive compounds including caffeine, CGA, diterpene alcohols, minerals such as potassium and magnesium, niacin and lignans [1]. Several studies have concluded that coffee has been inversely associated with diabetes risk and CRP levels $[9,10]$. However, caffeine in higher concentrations has been associated with acute increases in blood pressure and adverse effects on arterial stiffness and endothelium dependent vasodilation [6]. Furthermore, long-term heavy coffee consumption has been associated with risk of hypertension and higher homocysteine levels [11]. Thus, it remains to be further studied if the association between coffee and homocysteine levels explains the J-shaped association. It is therefore possible that with low and moderate coffee consumption, beneficial effects may be greater, whereas with heavy coffee consumption, negative effects may counterbalance the positive ones.

To our knowledge, based on a large sample of healthy subjects, this prospective study is one of the few in the literature that suggests a J-shaped association between coffee consumption and the risk of developing CVD [2, 4-6]. Until recently case-control studies have reported a J-shaped relation between coffee consumption and the risk of CVD [10,32]. In accordance with our findings the Greek CARDIO2000 case-control study examined the association between coffee consumption and the risk of developing acute coronary syndromes based on a large sample of coronary patients and CVD-free 
controls. After controlling for the presence of known CVD confounders, the analysis raised a J-shaped association between the quantity of coffee consumed per day and the risk of developing acute coronary syndromes. Moderate coffee consumption ( $<300 \mathrm{ml}$ coffee/d), was associated with a lower coronary risk, whereas heavy or very heavy consumption $(>300 \mathrm{ml} / \mathrm{d}$ ) was associated with a significant increase in risk [32]. This result may explain partially the contradictory results reports by earlier studies.

Although many studies in the past have attempted to assess the role of coffee consumption on CVD incidence, the findings remain inconsistent mainly as a result of the different study designs. With the exception of few studies, a recent meta-analysis of prospective cohort studies has concluded that moderate coffee (3-5 cups/d) consumption was inversely significantly associated with CVD risk, while heavy consumption was not, demonstrating a non-linear U-shaped association [6]. However, residual confounding by smoking may have biased the association for heavy coffee drinkers (higher prevalence of cigarette smoking) upward. The U- or J-shaped association could be attributed to the coffee's preparation: at higher intakes of coffee prepared, the intake of atherogenic coffee lipids may outweigh the potentially beneficial micronutrient compounds of coffee. Cohort studies generally showed a null association. Differences among studies in sample sizes, the characteristics of the study populations, the assessment methods for coffee consumption, and the statistical adjustments may have contributed to divergent results [33]. Since the true association between coffee consumption and CVD risk is likely to be modest and non linear, the differences in coffee assessments and covariate adjustments may result in changing the magnitude and even the direction of the associations and thus lead to different conclusions.

The major strengths of the present study are its prospective design, the large sample size from general population and a long period of follow up (10-years), which allowed us to assess the incidence of several outcomes. It is the first prospective study suggesting a J-shaped association between coffee consumption and 10-year CVD incidence, compared to case-control studies where recall biases affect their results. Another advantage of our study was that we conducted a first-event dose-response analysis which provides a comprehensive description of the shape of low/moderate and heavy coffee consumption that can be proved to be cardioprotective or detrimental for cardiovascular health. Particularly, our study is one of the few that extracted significant protective results for minimum daily coffee quantities reaching barely 1-2 cups of coffee that can contribute to the CVD prevention. Furthermore, our results remained significant when potential CVD confounders were taken into consideration among participants with or without the MetS; a condition that poses individuals by default at high CVD risk. In this work it was confirmed that low and moderate coffee consumption may reduce CVD risk among people at low risk, whereas focusing on high CVD risk individuals (e.g. with MetS) the protective effect of this habit seems to disappear. This carries a specific health message, since the adoption of dietary habits are more likely to reflect real practices of generally healthy individuals trying to reverse the results of several decades of oxidative stress.

\subsection{Limitations}

However, some limitations of the study should be acknowledged. Firstly, coffee consumption was assessed by FFQ at the time of recruitment and relied on participants' self report even though, results from dietary validation studies suggest that the use of FFQ for coffee self-report assessment is highly reproducible and agrees well with assessments using diet records [34]. Secondly, the number of cases is relatively few, but according to our statistical power analysis a-posteriori the achieved power was enough to evaluate the observed associations in our results. Furthermore, some participants may have changed their coffee habits during the long follow-up of 10 years, but the same research methodology has been widely used in prospective studies, thus, the results are comparable. As previously described, filtering may alter coffee's metabolic effects. The method of coffee preparation was not assessed in our study in our study population, and we were therefore not able to study whether the association between coffee consumption and CVD depends on the filtering of coffee. Next, we did not include information of decaffeinated coffee drinking which was also recorded, but not used in the analyses because of the very small number of participants reported drinking this type of coffee $(n=47)$.

\subsection{Conclusions}

Our findings provide an important public message, since moderate coffee appears to be beneficial on long-term CVD prevention, whereas heavy consumption is associated with significant increase of the 10-year CVD risk. The present study suggests a J-shaped coffee-CVD association that may partially explain the contradictory findings reported by previous studies. Interestingly, none of the inflammatory factors, speculated to mediate the coffee CVD association, seemed to modify significantly the association we observed. Finally, our findings that among subjects with MetS and hence after several years of exposure to oxidative stress, the protective effect of low to moderate coffee consumption against CVD was lost, suggests that it can only have such beneficial/preventive effects if such a habit is acquired at a relatively young age.

\section{Acknowledgements}

The authors would like to thank the ATTICA study group of investigators: Yannis Skoumas, Natasa Katinioti, Labros Papadimitriou, Constantina Masoura, Spiros Vellas, Yannis Lentzas, Manolis Kambaxis, Konstanitna Paliou, Vassiliki Metaxa, Agathi Ntzouvani, Dimitris Mpougatas, Nikolaos Skourlis, Christina Papanikolaou, Aikaterini Kalogeropoulou, Evangelia Pitaraki, Alexandros Laskaris, Mihail Hatzigeorgiou and Athanasios Grekas, Eleni Kokkou for either assistance in the initial physical examination and follow-up evaluation, Efi Tsetsekou for her assistance in psychological evaluation and follow-up evaluation, as well as laboratory team: Carmen Vassiliadou and George Dedousis (genetic 
analysis), Marina Toutouza-Giotsa, Constantina Tselika and Sia Poulopouloou (biochemical analysis) and Maria Toutouza for the database management.

\section{Conflict of interest}

The authors declare that they have no conflict of interest.

\section{Contributors}

Georgia-Maria Kouli had the concept of the paper, performed data analyses and interpreted the results. Demosthenes Panagiotakos, Christina Chrysohoou, Ekavi Georgousopoulou, contributed by providing the design of the study, critically reviewed the paper and approved the final version, Adela Zana, Constantine Tsigos, Dimitrios Tousoulis, Christodoulos Stefanadis and Christos Pitsavos critically reviewed the paper. All authors approved the final version.

Funding: D.B. Panagiotakos and E. Georgousopoulou received research grants by Coca-Cola SA. The ATTICA Study has been funded by research grants from the Hellenic Cardiology Society and the Hellenic Atherosclerosis Society.

\section{References}

1. O'Keefe JH, Bhatti SK, Patil HR, DiNicolantonio JJ, Lucan SC, Lavie CJ (2013) Effects of habitual coffee consumption on cardiometabolic disease, cardiovascular health, and all-cause mortality. J Am Coll Cardiol 17;62:1043-51. doi: 10.1016/j.jacc.2013.06.035.

2. Crippa A, Discacciati A, Larsson SC, Wolk A, Orsini N (2014) Coffee consumption and mortality from all causes, cardiovascular disease, and cancer: a dose-response meta-analysis. Am J Epidemiol 180:763-75. doi:10.1093/aje/kwu194.

3. Lopez-Garcia E, van Dam RM, Qi L, Hu FB (2006) Coffee consumption and markers of inflammation and endothelial dysfunction in healthy and diabetic women. Am J Clin Nutr 84:88893.

4. Liu J, Sui X, Lavie CJ, Hebert JR, Earnest CP, Zhang J, Blair SN (2013) Association of coffee consumption with all-cause and cardiovascular disease mortality. Mayo Clin Proc 88:1066-74. doi: 10.1016/j.mayocp.2013.06.020.

5. Floegel A, Pischon T, Bergmann MM, Teucher B, Kaaks R, Boeing H (2012) Coffee consumption and risk of chronic disease in the European Prospective Investigation into Cancer and Nutrition (EPIC)-Germany study. Am J Clin Nutr 95:901-8. doi: 10.3945/ajcn.111.023648.

6. Ding M, Bhupathiraju SN, Satija A, van Dam RM, Hu FB (2014) Long-term coffee consumption and risk of cardiovascular disease: a systematic review and a dose-response meta-analysis of prospective cohort studies. Circulation 129:643-59. doi: 10.1161/CIRCULATIONAHA.113.005925.

7. Zampelas A, Panagiotakos DB, Pitsavos C, Chrysohoou C, Stefanadis C (2004) Associations between coffee consumption and inflammatory markers in healthy persons: the ATTICA study. Am J Clin Nutr 80:862-7.

8. Yamashita K, Yatsuya H, Muramatsu T, Toyoshima H, Murohara T, Tamakoshi K (2012) Association of coffee consumption with serum adiponectin, leptin, inflammation and metabolic markers in Japanese workers: a cross-sectional study. Nutr Diabetes 2:e33. doi: 10.1038/nutd.2012.6.

9. Andersen LF, Jacobs DR Jr, Carlsen MH, Blomhoff R (2006) Consumption of coffee is associated with reduced risk of death attributed to inflammatory and cardiovascular diseases in the Iowa Women's Health Study. Am J Clin Nutr 83:1039-46.

10. Vlachopoulos C, Panagiotakos D, Ioakeimidis N, Dima I, Stefanadis C (2005) Chronic coffee consumption has a detrimental effect on aortic stiffness and wave reflections. Am J Clin Nutr 81:1307-12.

11. Mesas AE, Leon-Muñoz LM, Rodriguez-Artalejo F, Lopez-Garcia E (2011) The effect of coffee on blood pressure and cardiovascular disease in hypertensive individuals: a systematic review and meta-analysis. Am J Clin Nutr 94:1113-26. doi: 10.3945/ajcn.111.016667.

12. Pitsavos C, Panagiotakos DB, Chrysohoou C, Stefanadis C (2003) Epidemiology of cardiovascular risk factors in Greece: aims, design and baseline characteristics of the ATTICA study. BMC Public Health 3:32.

13. Katsouyanni K, Rimm EB, Gnardellis C, Trichopoulos D, Polychronopoulos E, Trichopoulou A (1997) Reproducibility and relative validity of an extensive semiquantitative food frequency questionnaire using dietary records and biochemical markers among Greek schoolteachers. Int J Epidemiol 26: S118-127.

14. Panagiotakos DB, Pitsavos C, Stefanadis C (2006) Dietary patterns: a Mediterranean diet score and its relation to clinical and biological markers of cardiovascular disease risk. Nutr Metab Cardiovasc Dis 16: 559-568.

15. Papathanasiou G, Georgoudis G, Papandreou M, Spyropoulos P, Georgakopoulos D, Kalfakakou V, Evangelou A. (2009) Reliability measures of the short International Physical Activity Questionnaire (IPAQ) in Greek young adults. Hellenic J Cardiol 50:283-294.

16. American Diabetes Association (1997) Report of the Expert Committee on the Diagnosis and Classification of Diabetes Mellitus. Diabetes Care 20: 1183-97.

17. Grundy SM, Brewer HB Jr., Cleeman JI, Smith SC Jr., Lenfant C (2004) Definition of metabolic syndrome: report of the National Heart, Lung, and Blood Institute/American Heart Association conference on scientific issues related to definition. Circulation 109:433-8. 
18. Bunker ML, McWilliams M (1979) Caffeine content of common beverages. J Am Diet Assoc 74: 2832.

19. Panagiotakos DB, Georgousopoulou EN, Pitsavos C, Chrysohoou C, Metaxa V, Georgiopoulos GA, Kalogeropoulou K, Tousoulis D, Stefanadis C; ATTICA Study group (2015) Ten-year (2002-2012) cardiovascular disease incidence and all-cause mortality, in urban Greek population: the ATTICA Study. Int J Cardiol 180:178-84. doi: 10.1016/j.ijcard.2014.11.206.

20. Zampelas A, Panagiotakos DB, Pitsavos C, Chrysohoou C, Stefanadis C (2004) Associations between coffee consumption and inflammatory markers in healthy persons: the ATTICA study. Am J Clin Nutr 80:862-7.

21. Georgousopoulou EN, Kouli GM, Panagiotakos DB, Kalogeropoulou A, Zana A, Chrysohoou C, Tsigos C, Tousoulis D, Stefanadis C, Pitsavos C (2016) Anti-inflammatory diet and 10-year (2002-2012) cardiovascular disease incidence: The ATTICA study. Int J Cardiol 222:473478. doi: $10.1016 /$ j.ijcard.2016.08.007.

22. Czernichow S, Vergnaud AC, Galan P, Arnaud J, Favier A, Faure H, Huxley R, Hercberg S, Ahluwalia N (2009) Effects of long-term antioxidant supplementation and association of serum antioxidant concentrations with risk of metabolic syndrome in adults. Am J Clin Nutr 90:329-35. doi: $10.3945 /$ ajcn.2009.27635.

23. Steinhubl SR (2008) Why have antioxidants failed in clinical trials? Am J Cardiol 101:14D-19D.

24. Valtueña S, Pellegrini N, Franzini L, Bianchi MA, Ardigò D, Del Rio D, Piatti

P, Scazzina F, Zavaroni I, Brighenti F (2008) Food selection based on total antioxidant capacity can modify antioxidant intake, systemic inflammation, and liver function without altering markers of oxidative stress. Am J Clin Nutr 87:1290-7.

25. Wyss-Coray T, Mucke L (2002) Inflammation in neurodegenerative disease--a double-edged sword. Neuron 35:419-32.

26. Vakkila J, Lotze MT (2004) Inflammation and necrosis promote tumour growth. Nat Rev Immunol 4:641-8.

27. Quinn MT, Gauss KA (2004) Structure and regulation of the neutrophil respiratory burst oxidase: comparison with nonphagocyte oxidases. J Leukoc Biol 76:760-81.

28. Masella R, Di Benedetto R, Varì R, Filesi C, Giovannini C (2005) Novel mechanisms of natural antioxidant compounds in biological systems: involvement of glutathione and glutathione-related enzymes. J Nutr Biochem 16:577-86.

29. Lockyer S, Rowland I, Spencer JP, Yaqoob P, Stonehouse W (2016) Impact of phenolic-rich olive leaf extract on blood pressure, plasma lipids and inflammatory markers: a randomised controlled trial. Eur J Nutr [online available ahead of print]

30. Agudelo-Ochoa GM, Pulgarín-Zapata IC, Velásquez-Rodriguez CM, Duque-Ramírez M, NaranjoCano M, Quintero-Ortiz MM, Lara-Guzmán OJ, Muñoz-Durango K (2016) Coffee Consumption Increases the Antioxidant Capacity of Plasma and Has No Effect on the Lipid Profile or Vascular Function in Healthy Adults in a Randomized Controlled Trial. J Nutr 146:524-31.

31. Chang WC, Chen CH, Lee MF, Chang T, Yu YM (2010) Chlorogenic acid attenuates adhesion molecules upregulation in IL-1beta-treated endothelial cells. Eur J Nutr 49:267-75. doi: 10.1007/s00394-009-0083-1.

32. Panagiotakos DB, Pitsavos C, Chrysohoou C, Kokkinos P, Toutouzas P, Stefanadis C (2003) The Jshaped effect of coffee consumption on the risk of developing acute coronary syndromes: the CARDIO2000 case-control study. J Nutr 133:3228-32.

33. Zulli A, Smith RM, Kubatka P, Novak J, Uehara Y, Loftus $H$, Qaradakhi T, Pohanka M, Kobyliak N, Zagatina A, Klimas J, Hayes A, La Rocca G, Soucek M, Kruzliak P (2016) Caffeine and cardiovascular diseases: critical review of current research. Eur J Nutr 55:1331-43. doi: 10.1007/s00394-016-1179-z.

34. Bohlscheid-Thomas S, Hoting I, Boeing H, Wahrendorf J (1997). Reproducibility and relative validity of energy and macronutrient intake of a food frequency questionnaire developed for the German part of the EPIC project. European Prospective Investigation into Cancer and Nutrition. Int J Epidemiol 26 Suppl 1:S71-81. 